\title{
Multiple Sclerosis
}

MRI is unquestionably the imaging modality of choice for evaluation of white matter diseases. Although a routine brain MR exam would suffice in many applications, specific protocols for multiple sclerosis have been developed to maximize its contribution. This unit presents one such protocol while recognizing the wide opportunity for variation depending on local clinical demand and personal preference.

\section{MULTIPLE SCLEROSIS}

The basic goal in the evaluation of the suspected multiple sclerosis is one of plaque identification followed by characterization. All demyelinating lesions exhibit $T_{2}$ prolongation, and so the exam begins with some form of $T_{2}$ weighting. The choice of such weighting, along with imaging plane, will be discussed. Although many approaches exist for plaque characterization, they generally include a search for enhancement as a measure of activity. Rather than being an all-or-none phenomenon, enhancement appears to be a matter of degree, and so several tricks have evolved to maximize detection of enhancement. These tricks, and their advantages and limitations, will be discussed. The parameters given here are derived from experience at $1.5 \mathrm{~T}$, and may need to be altered depending on the field strength and the equipment manufacturer. The entire procedure generally takes $\sim 45$ min.

Table A5.1.1 summarizes the six sequences which comprise the basic protocol along with two optional sequences. Table A5.1.2 lists the hardware necessary to perform the procedure. Next, stepwise instructions for performing the protocol are provided. The protocol is not particularly demanding on scanner hardware and is of the sort which may be well performed on most clinical systems.

NOTE: Be sure that technologists and nurses have immediate access to any equipment such as crash carts or oxygen that may be necessary in the event of an emergency.

\section{Materials}

Normal saline $(0.9 \% \mathrm{NaCl})$, sterile

Extravascular contrast agent (e.g., Magnevist, Omniscan, or Prohance)

Table A5.1.1 Basic Multiple Sclerosis Protocol ${ }^{a}$

\begin{tabular}{ll}
\hline Type of weighting and sequence & $\begin{array}{c}\text { Imaging } \\
\text { plane }\end{array}$ \\
\hline 1. Pilot scan (scout) & - \\
2. $T_{1}$-weighted spin echo & Sagittal \\
3. Fast FLAIR & Sagittal \\
4. Dual echo PD/ $T_{2}$-weighted TSE/FSE & Transverse \\
5. $T_{1}$-weighted spin echo & Transverse \\
6. Post-gadolinium $T_{1}$-weighted spin echo & Transverse \\
Optional sequences & \\
7. Dual echo PD/ $T_{2}$-weighted CSE & Transverse \\
8. $T_{1}$-weighted MT spin echo & Transverse \\
${ }^{a}$ Abbreviations: TSE, turbo spin echo; FSE, fast spin echo; CSE, con- \\
ventional spin echo; MT, magnetization transfer; FLAIR, fluid attenu- \\
ated inversion recovery; PD, proton density.
\end{tabular}

Contributed by Danial K. Hallam

Current Protocols in Magnetic Resonance Imaging (2001) A5.1.1-A5.1.15

Copyright $\odot 2001$ by John Wiley \& Sons, Inc.
BASIC

PROTOCOL
Miscellaneous Brain Pathology 
Table A5.1.2 Equipment Parameters for MRI In Multiple Sclerosis

\begin{tabular}{ll}
\hline Coil type & Quadrature head coil \\
Gradient coil strength & Whatever the system permits \\
Cardiac gating & No \\
Peripheral monitoring & For safety only \\
Respiratory gating & No \\
Respirator & If required by patient \\
Oxygen & If required by patient \\
Motion cushions & Useful \\
\hline
\end{tabular}

\section{Setup equipment and patient}

1. Interview (screen) the patient to ensure that he or she has no contraindications such as cardiac pacemakers or other implants containing ferromagnetic materials. Also be sure to find out if the patient has any health conditions that may require the presence of special emergency equipment or other precautions during the scanning procedure.

Generally standard screening forms are used for all patients scanned in a magnetic resonance system.

The presence of any ferromagnetic metals may be a health hazard to the patient when he or she is inside the magnet, and will also affect the imaging. If in doubt as to the exact composition of the items, it is best to exclude patients with any metal implants; see Shellock (1996) for discussion of what implants may be safely scanned using magnetic resonance.

Patients may be accompanied into the magnet room by a friend or family member, who can sit in the room during the scan and comfort the patient as needed. This companion must be screened as well to ensure the absence of loose metal objects on the body or clothing.

2. If the procedure is a research protocol, have the patient sign any necessary consent form.

3. Have the patient remove all jewelry and change into a gown to eliminate any metal that might be found in clothing.

4. Have the patient wash off mascara or other makeup to avoid local tissue heating and image artifacts.

5. Inform the patient about what will occur during the procedure, what he or she will experience while in the magnet, and how to behave, including the following:

a. If earphones or headphones are used to protect the ears from the loud sounds produced by the gradients, the patient will be asked to wear these, but will be able to communicate with you at any time during the imaging.

b. The patient will be given a safety squeeze-bulb or similar equipment to request assistance at any time (demonstrate how this works).

c. For good results the patient should be instructed not to talk unless absolutely necessary and to avoid or minimize swallowing and other movements during each scan-i.e., as long as the banging sounds continue. Between scans, talking and swallowing are acceptable in most cases, but should be avoided when comparative positional studies are being performed; the patient will be informed when this is the case.

d. Nevertheless, the patient may call out at any time if he or she feels it necessary.

6. Have the patient mount onto the table. Set up any triggering devices or other monitoring equipment that is to be used either before or just after the patient lies down. 
7. Center the patient in the head coil. Make sure that the head and neck are constrained to limit motion, especially if high-resolution scans are to be run.

Generally the patient's head is fixed so that the head is horizontal (not tilted) and the neck and head lie along the axis of the patient table; other positions may be appropriate depending on the needs at hand.

8. If needed, place a pillow or other support under the knees to make the patient more comfortable.

9. Establish an intravenous (i.v.) line from which the contrast agent can be injected, and attach this line securely to the patient so that movement into or out of the magnet will not pull at the patient's arm.

This step may be performed prior to entering the magnet room if necessary to save scanner time.

It is preferable to insert the line prior to imaging and to leave the patient in the magnet, with no intervening motion between the scans run before contrast agent injection and those run after injection.

10. Use the centering light to position the patient's nasion and put him or her into the center of the magnet.

Once this step has been performed, so long as the patient does not move on the table, the table itself can be moved and then replaced in the same position as before without jeopardizing the positioning of one scan relative to another.

11. If the patient is unable to hold still, provide an appropriate sedative.

\section{Sequence 1: Rapid three-plane positioning pilot}

12. To validate the patient's position, run the system's pilot (or scout) scan to ensure correct location of the head in three dimensions, using the imaging sequence given in Table A5.1.3 or similar parameters.

Table A5.1.3 Parameters for Pilot Scan (Scout; Sequence 1)

\begin{tabular}{ll}
\hline Patient position & Supine \\
Scan type & Gradient echo \\
Imaging plane (orientation) & 3 planes \\
Central slice or volume center & Run initially at magnet isocenter ${ }^{a}$ \\
Echo time $\left(T_{\mathrm{E}}\right)$ & Minimum \\
Repeat time $\left(T_{\mathrm{R}}\right)$ & Minimum \\
Flip angle (FA) & $20^{\circ}$ \\
Fields of view $\left(\mathrm{FOV}, \mathrm{FOV}_{\mathrm{y}}\right)$ & $300 \mathrm{~mm}, 300 \mathrm{~mm}$ \\
Resolution $(\Delta x, \Delta y)$ & $1.17 \mathrm{~mm}, 1.56 \mathrm{~mm}$ \\
Number of data points collected $\left(N_{\mathrm{x}}, N_{\mathrm{y}}\right)$ & 256,192 \\
Display matrix $\left(D_{\mathrm{x}}, D_{\mathrm{y}}\right)$ & 256,256 \\
Slice thickness $(\Delta z)$ & $5 \mathrm{~mm}$ \\
Number of slices & 3, one in each of 3 cardinal planes \\
Slice gap & Not applicable \\
Number of acquisitions $\left(N_{\text {acq }}\right)$ & 1 \\
Swap read and phase encoding & No \\
Slice locations & Not applicable \\
Saturation pulses & Not applicable \\
Scan time & $\sim 10$ sec \\
\hline${ }_{\text {If }}$ the pilot scan shows the patient's head to be significantly off-center, then it may be helpful to \\
reposition the patient and repeat the pilot scan. & \\
\end{tabular}


This sequence usually consists of three orthogonal planes to allow localization. The images are often also used later to determine where to place the saturation pulses and to set up total coverage of the volume of interest.

\section{Sequence 2: $T_{1}$-weighted spin echo sagittal}

13. Using images generated in sequence 1, sagittal images are planned to provide whole brain coverage. Set the parameters as indicated in Table A5.1.4.

The scan is best positioned first on the transverse pilot scan. Some angulation may be needed to provide for true sagittal anatomic imaging. The coverage provided should then be checked on the sagittal scout. This sequence is used to accurately position subsequent scans. It provides excellent visualization of midline structures.

\section{Sequence 3: Fast FLAIR sagittal}

14. Set parameters for fast FLAIR sagittal as indicated in Table A5.1.5.

The scan is positioned just as in sequence 2. The sequence provides superior demonstration of $T_{2}$-weighted bright lesions, especially in the supratentorial compartment. The suppression of bright CSF (cerebrospinal fluid) afforded by the FLAIR technique demonstrates lesions adjacent to CSF that would otherwise be poorly visualized. The use of the sagittal plane for the study provides detailed visualization of lesions of the corpus callosum (Figure A5.1.1). Such lesions are not only common in multiple sclerosis but are judged to be fairly specific for this diagnosis (Gean-Marton et al., 1991).

\section{Sequence 4: Dual echo PD/T $T_{2}$-weighted transverse}

15. The $T_{1}$-weighted sagittal image is used to position transverse scans. Set parameters for the $\mathrm{PD} / T_{2}$-weighted dual echo transverse sequence as given in Table A5.1.6.

The scan is positioned graphically to provide whole brain coverage. The slices should be angled according to an institutional standard for transverse images. The author recommends using the "AC-PC" line as such a standard. Here, the anterior and posterior commissures are identified on the $T_{1}$-weighted sagittal midline image (Fig. A5.1.2). The slices of all transverse scans are positioned parallel to a line drawn between the two landmarks. The sequence provides excellent visualization of the whole brain. The transverse plane is the most useful for evaluating demyelinating lesions involving the brainstem (Figure A5.1.3). Proton-density weighting optimally visualizes lesions on the surface of the brainstem, whereas the combination of $T_{2}$-weighted and proton-density-weighted images is best for deeper brainstem lesions. (FLAIR, although superior for evaluation of much intracranial pathology, especially that adjacent to CSF, has proven less sensitive to posterior fossa lesions; Bastianello et al., 1997; Tubridy et al., 1998).

\section{Sequence 5: $T_{1}$-weighted spin echo transverse}

16. Set parameters for the $T_{1}$-weighted spin echo transverse sequence as indicated in Table A5.1.7 and position identically to sequence 4 , dual PD/ $T_{2}$-weighted transverse sequence.

The scan provides visualization of $T_{1}$-weighted hypointense demyelinating lesions. Such lesions have been shown to correlate better with the degree of disability than $T_{2}$-weighted lesions (van Walderveen et al., 1995; Truyen et al., 1996; Giugni et al., 1997). The $T_{1}$-weighted spin echo transverse image provides the appropriate comparison for evaluation of contrast enhancement. Some demyelinating plaques, especially those that are chronic, will exhibit borders of intrinsic $T_{1}$-weighted hyperintensity (generally thought to be evidence of myelin breakdown products). Without the benefit of a pre-contrast scan performed identically to that post-contrast, differentiation of such areas of intrinsic $T_{1}$-weighted hyperintensity from true enhancement may not be possible.

17. Inject the contrast agent, then flush the intravenous line with $10 \mathrm{ml}$ saline. It is preferable to perform the contrast injection while leaving the patient in the magnet.

A dose of $0.1 \mathrm{mmol} / \mathrm{kg}$ of contrast agent is usually given. Triple dose $(0.3 \mathrm{mmol} / \mathrm{kg}) \mathrm{may}$ be used to improve demonstration of enhancement. 
Sequence 6: $T_{1}$-weighted spin echo post-contrast transverse

18. Simply repeat sequence 5 after injecting contrast using the parameters as given in Table A5.1.7.

Gadolinium administration is not generally necessary for the diagnosis of multiple sclerosis but it is very helpful for assessment of disease activity, as only acute lesions enhance. Several techniques exist which are meant to provide more sensitive evaluation of the breakdown of blood-brain barrier indicated by enhancement. Use of triple the usual dose

Table A5.1.4 Parameters for $T_{1}$-Weighted Spin Echo Sagittal (Sequence 2)

\begin{tabular}{ll}
\hline Patient position & Supine \\
Scan type & Spin echo \\
Imaging plane (orientation) & Sagittal \\
Central slice or volume center & Midline \\
Echo time $\left(T_{\mathrm{E}}\right)$ & $14 \mathrm{msec}$ \\
Repeat time $\left(T_{\mathrm{R}}\right)$ & $600 \mathrm{msec}$ \\
Flip angle $(\mathrm{FA})$ & $90^{\circ}$ \\
Fields of view $\left(\mathrm{FOV}, \mathrm{x}, \mathrm{FOV}_{\mathrm{y}}\right)$ & $230 \mathrm{~mm}, 230 \mathrm{~mm}$ \\
Resolution $(\Delta x, \Delta y)$ & $1.2 \mathrm{~mm}, 0.9 \mathrm{~mm}$ \\
Number of data points collected $\left(N_{\mathrm{x}}, N_{\mathrm{y}}\right)$ & 192,256 \\
Slice thickness $(\Delta z)$ & $5 \mathrm{~mm}$ \\
Number of slices & $19-21$ \\
Slice gap (distance factor) & $1.5 \mathrm{~mm}(0.30)$ \\
Number of acquisitions $\left(N_{\mathrm{acq}}\right)$ & 1 \\
Read direction & Cranio-caudal \\
Saturation pulses & Inferior may be used \\
Scan time & $\sim 2$ min \\
\hline
\end{tabular}

Table A5.1.5 Parameters for Fast FLAIR Sagittal (Sequence 3)

\begin{tabular}{ll}
\hline Patient position & Supine \\
Scan type & Fast FLAIR \\
Imaging plane (orientation) & Sagittal \\
Central slice or volume center & Midline \\
Echo time $\left(T_{\mathrm{E}}\right)^{a}$ & $100-140 \mathrm{msec}$ \\
Echo train length (ETL) (turbo factor) $\left.^{(}\right)$ & $7-11$ \\
Repeat time $\left(\mathrm{T}_{\mathrm{R}}\right)^{a}$ & $8,000-10,000 \mathrm{msec}$ \\
Inversion time $\left(\mathrm{T}_{\mathrm{I}}\right)^{a}$ & $2300 \mathrm{msec}$ \\
Flip angle $(\mathrm{FA})$ & $180^{\circ}$ \\
Fields of view $\left(\mathrm{FOV}, \mathrm{x}, \mathrm{FOV}_{\mathrm{y}}\right)$ & $230 \mathrm{~mm}, 230 \mathrm{~mm}$ \\
Resolution $(\Delta x, \Delta y)$ & $0.9 \mathrm{~mm}, 0.9 \mathrm{~mm}$ \\
Number of data points collected $\left(N_{\mathrm{x}}, N_{\mathrm{y}}\right)$ & 256,256 \\
Slice thickness $(\Delta z)$ & $4-5 \mathrm{~mm}$ \\
Number of slices & 21 \\
Slice gap & $1.5 \mathrm{~mm}$ (or run twice and \\
& interleave with zero gap) \\
Number of acquisitions $\left(N_{\text {acq }}\right)$ & 1 \\
Read direction & Cranio-caudal \\
Saturation pulses & Inferior may be used \\
Scan time & $\sim 4$ min \\
\hline${ }^{a}$ Optimum choice of parameters for fast FLAIR varies significantly with manufacturer and scanner \\
limitations. For a review of parameter optimization in fast FLAIR (see Rydberg et al., 1995).
\end{tabular}

Miscellaneous Brain Pathology

A5.1.5 
of gadolinium is likely the most powerful technique (Bastianello et al., 1998). Please see discussion of augmenting enhancement under Commentary.

\section{Sequence 7: Dual echo PD/T $T_{2}$-weighted conventional spin echo (optional)}

19. Sequence 7 may be used in lieu of sequence 4 using parameters in Table A5.1.8.

The sequence will require more scanning time than the FSE/TSE and a larger slice gap is required to provide whole brain imaging. It is comparable in lesion detection to sequence 4 (see discussion in the Commentary).

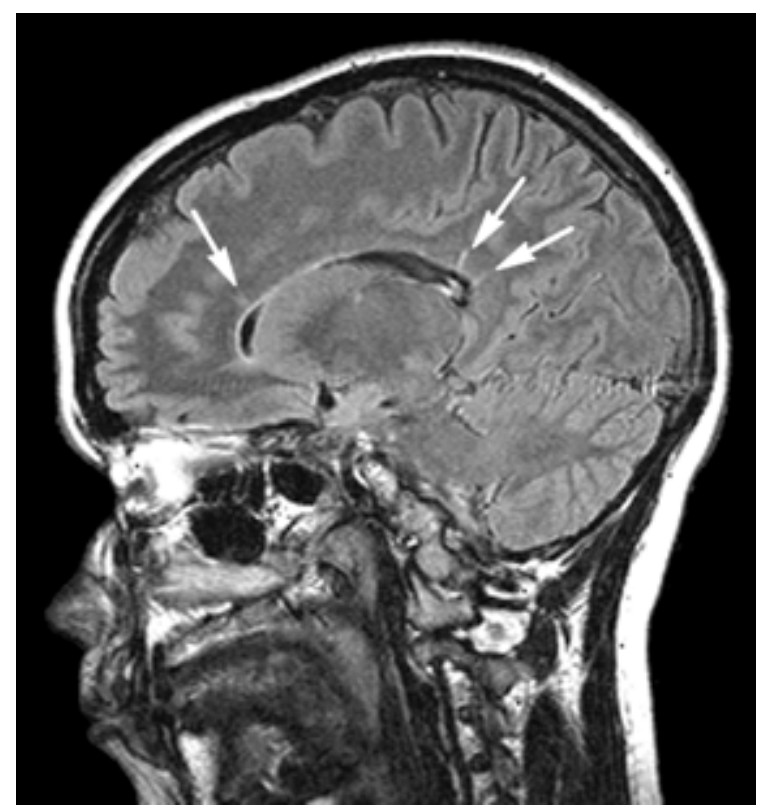

Figure A5.1.1 Fast FLAIR sagittal provides optimal visualization of small callosal multiple sclerosis lesions sometimes referred to as subependymal striations.

Table A5.1.6 Parameters for Dual Echo PD/ $T_{2}$-Weighted TSE/FSE Transverse (Sequence 4)

Patient position

Scan type

Imaging plane (orientation)

Central slice or volume center

Echo time $\left(T_{\mathrm{E}}\right)$

Echo train length (ETL) (turbo factor)

Repeat time $\left(T_{\mathrm{R}}\right)$

Flip angle (FA)

Fields of view $\left(\mathrm{FOV}_{\mathrm{x}}, \mathrm{FOV}_{\mathrm{y}}\right)$

Resolution $(\Delta x, \Delta y)$

Number of data points collected $\left(N_{\mathrm{x}}, \mathrm{N}_{\mathrm{y}}\right)$

Slice thickness $(\Delta z)$

Number of slices

Slice gap (distance factor)

Number of acquisitions $\left(N_{\mathrm{acq}}\right)$

Read direction

Saturation pulses

Scan time
Supine

TSE/FSE

Transverse

Position for whole brain coverage

14-17 msec for PD-weighted image and 85-102 msec for

$T_{2}$-weighted image

$5-8$

2500-3000 msec

$90^{\circ}$

$180 \mathrm{~mm}, 230 \mathrm{~mm}$

$0.94 \mathrm{~mm}, 0.90 \mathrm{~mm}$

192,256

$5 \mathrm{~mm}$

23

$1.5 \mathrm{~mm}(0.30)$

1

Anterior to posterior

Inferior may be used

$\sim 3 \mathrm{~min}$ 


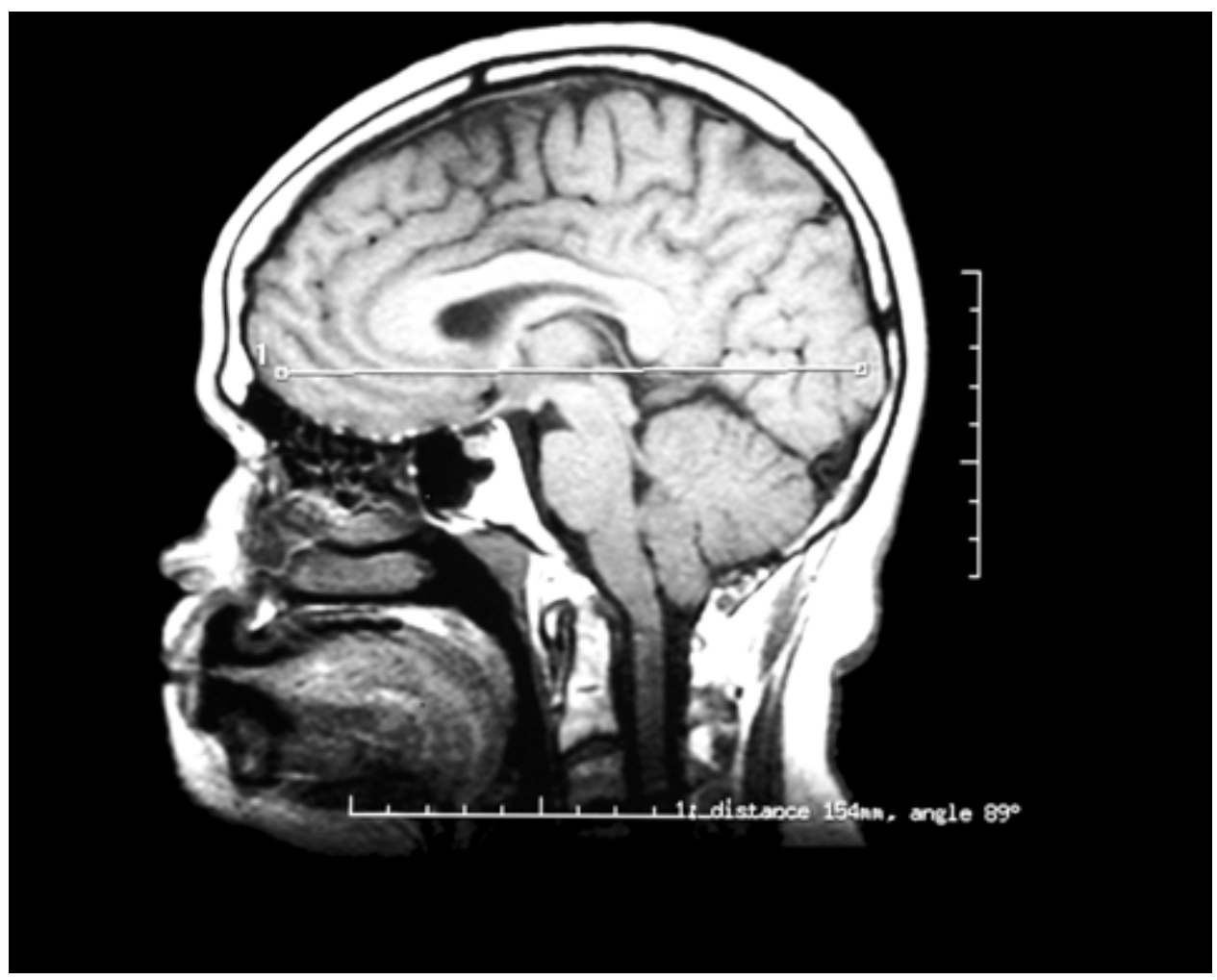

Figure A5.1.2 Midline $T_{1}$-weighted sagittal image demonstrates placement of AC-PC line to be used to position transverse images.

A

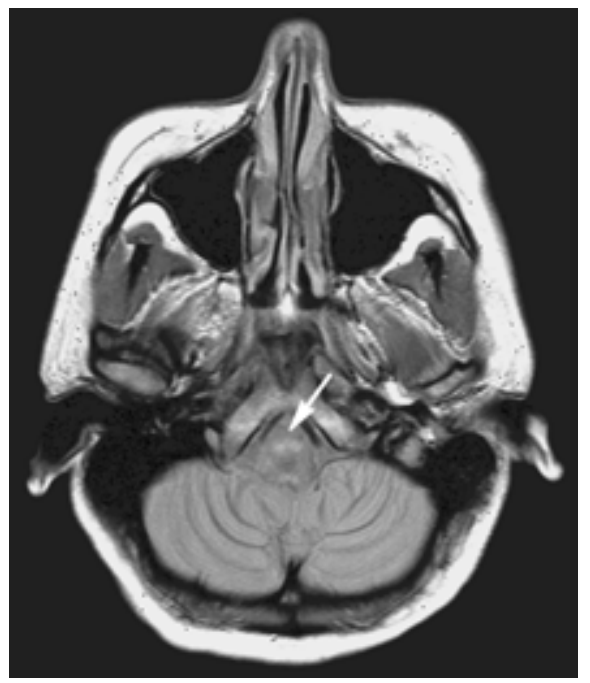

B

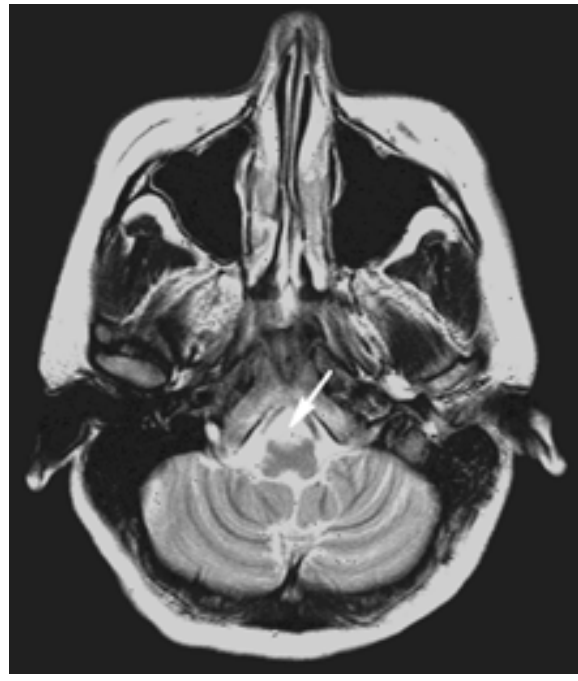

Figure A5.1.3 Combination of $\operatorname{PD}(\mathbf{A})$ and $T_{2}$-weighted $(\mathbf{B})$ transverse images demonstrates even very small brainstem lesions as shown here in the ventral medulla. Such lesions are important for several reasons. Brainstem lesions are frequently clinically apparent and imaging confirmation is generally preferred by the clinician. Also, multiple sclerosis brainstem lesions are often located along the cisternal or ventricular surface-a feature atypical for senescent/ischemic lesions. 
20. Run sequence 8 using parameters in Table A5.1.9 and position similarly to other transverse sequences.

The increased time required for the magnetization transfer pulse increases $T_{R}$ and reduces the maximum number of slices. An increase in number of acquisitions is generally needed for satisfactory image quality.

Table A5.1.7 Parameters for $T_{1}$-Weighted Spin Echo Transverse (Sequences 5 and 6)

\begin{tabular}{ll}
\hline Patient position & Supine \\
Scan type & Spin echo \\
Imaging plane (orientation) & Transverse \\
Central slice or volume center & Position for whole brain coverage \\
Echo time $\left(T_{\mathrm{E}}\right)$ & $14 \mathrm{msec}$ \\
Repeat time $\left(T_{\mathrm{R}}\right)$ & $600 \mathrm{msec}$ \\
Flip angle $(\mathrm{FA})$ & $90^{\circ}$ \\
Fields of view $(\mathrm{FOV}, \mathrm{FOV}$ & $\mathrm{F})$ \\
Resolution $(\Delta x, \Delta y)$ & $180 \mathrm{~mm}, 230 \mathrm{~mm}$ \\
Number of data points collected $\left(N_{\mathrm{x}}, N_{\mathrm{y}}\right)$ & $0.94 \mathrm{~mm}, 0.90 \mathrm{~mm}$ \\
Slice thickness $(\Delta z)$ & 192,256 \\
Number of slices & $5 \mathrm{~mm}$ \\
Slice gap (distance factor) & 23 \\
Number of acquistitions $\left(N_{\mathrm{acq}}\right)$ & $1.5 \mathrm{~mm}(0.30)$ \\
Read direction & 1 \\
Saturation pulses & Anterior to posterior \\
Scan time & Inferior may be used \\
\hline
\end{tabular}

Table A5.1.8 Parameters for Dual Echo PD/ $T_{2}$-Weighted Conventional Spin Echo (Sequence 7)

Patient position

Scan type

Imaging plane (orientation)

Central slice or volume center

Echo time $\left(T_{\mathrm{E}}\right)$

Repeat time $\left(T_{\mathrm{R}}\right)$

Flip angle (FA)

Fields of view $\left(\mathrm{FOV}_{\mathrm{x}}, \mathrm{FOV}_{\mathrm{y}}\right)$

Resolution $(\Delta x, \Delta y)$

Number of data points collected $\left(N_{\mathrm{x}}, N_{\mathrm{y}}\right)$

Slice thickness $(\Delta z)$

Number of slices

Slice gap (distance factor)

Number of acquisitions $\left(N_{\text {acq }}\right)$

Read direction

Saturation pulses

Scan time
Supine

Conventional spin echo

Transverse

Position for whole brain coverage

$30 \mathrm{msec}$ for PD-weighted image and $80 \mathrm{msec}$ for $T_{2}$-weighted

image

$2500 \mathrm{msec}$

$90^{\circ}$

$180 \mathrm{~mm}, 230 \mathrm{~mm}$

$0.94 \mathrm{~mm}, 0.90 \mathrm{~mm}$

192, 256

$5 \mathrm{~mm}$

20

$2.5 \mathrm{~mm}(0.50)$

$0.75^{a}$

Anterior to posterior Inferior may be used $\sim 5 \mathrm{~min}$

${ }^{a}$ Refers to 3/4 NEX imaging, also known as partial Fourier imaging — an option available on many clinical scanners. Partial Fourier imaging reduces imaging time by exploiting the inherent symmetry of $k$-space; essentially, fewer phase encoding steps are used to provide the same spatial resolution. The time saved is at the expense of signal to noise. 
Magnetization transfer imaging has multiple potential uses in everyday clinical imaging of multiple sclerosis and still more in the investigational arena. In routine use, MT may be helpful in improving specificity in the diagnosis (Mehta et al., 1996) of periventricular demyelinating lesions (Fig. A5.1.4). Also, it is one of the tricks used to augment enhancement. Both of these issues are discussed in further detail under Commentary.

Table A5.1.9 Parameters for $T_{1}$-Weighted Magnetization Transfer Spin Echo Transverse (Sequence 8)

\begin{tabular}{ll}
\hline Patient position & Supine \\
Scan type & Spin echo with MT pulse \\
Imaging plane (orientation) & Transverse \\
Central slice or volume center & Position for whole brain coverage \\
Echo time $\left(T_{\mathrm{E}}\right)$ & $15 \mathrm{msec}$ \\
Repeat time $\left(T_{\mathrm{R}}\right)$ & $715 \mathrm{msec}$ \\
Flip angle $(\mathrm{FA})$ & $90^{\circ}$ \\
Fields of view $(\mathrm{FOV}, \mathrm{\textrm {x }}, \mathrm{FOV} \mathrm{y})$ & $180 \mathrm{~mm}, 230 \mathrm{~mm}$ \\
Resolution $(\Delta x, \Delta y)$ & $0.94 \mathrm{~mm}, 0.90 \mathrm{~mm}$ \\
Number of data points collected $\left(N_{\mathrm{x}}, N_{\mathrm{y}}\right)$ & 192,256 \\
Slice thickness $(\Delta z)$ & $5 \mathrm{~mm}$ \\
Number of slices & 19 \\
Slice gap (distance factor) & $1.5 \mathrm{~mm}(0.30)$ \\
Number of acquisitions $\left(N_{\mathrm{acq}}\right)$ & 2 \\
Read direction & Anterior to posterior \\
Saturation pulses & Inferior may be used \\
Scan time & $\sim 5$ min \\
\hline
\end{tabular}

A

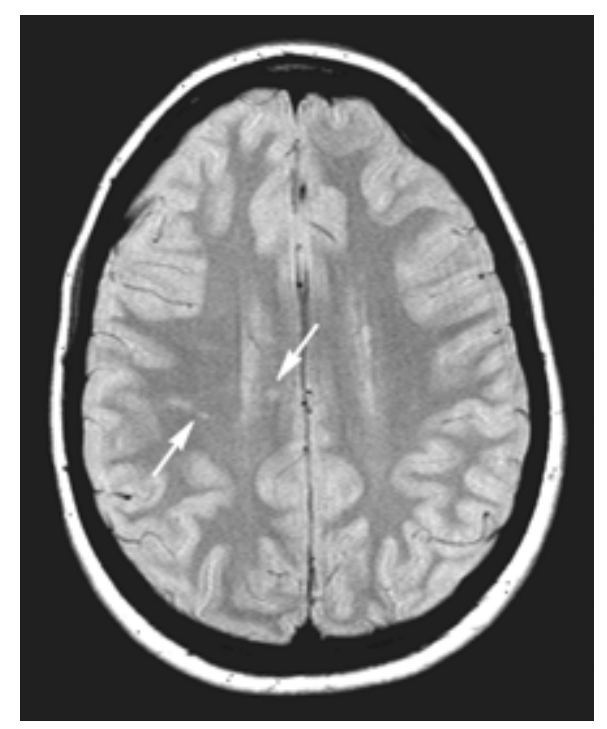

B

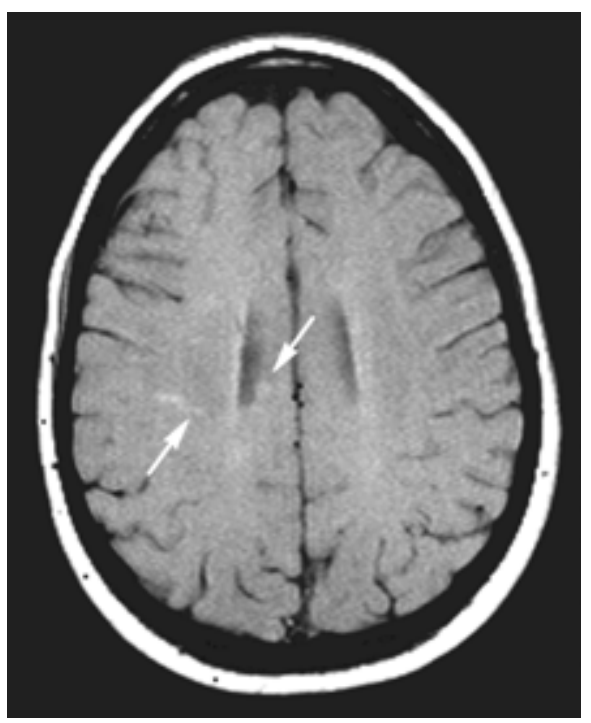

Figure A5.1.4 $\quad T_{2}$-weighted TSE transverse $(\mathbf{A})$ and $T_{1}$-weighted MT spin echo transverse (B) images demonstrate multiple callosal and periventricular lesions in this patient with multiple sclerosis. The increased signal on the $T_{1}$-weighted MT sequence is seen in some but not all demyelinating lesions. It is not generally seen with senescent/ischemic lesions and so is somewhat specific for demyelinating disease.

Miscellaneous Brain Pathology

A5.1.9 


\section{COMMENTARY}

\section{Background Information}

Owing to its high incidence, chronicity, and tendency to attack young adults, multiple sclerosis is one of the most important neurologic diseases. Although the exact cause is yet to be elucidated, autoimmune mechanisms and genetic susceptibility are generally regarded to be central. Prevalence increases with latitude, and clear racial differences exist with Caucasians at greatest risk. In the United States, individuals have a one in one thousand risk of developing multiple sclerosis during their lifetime. Annual costs related to the disease, including loss of productivity, rehabilitation, and medical care, have been estimated at over 2.5 billion dollars.

MRI has fundamentally altered the evaluation of this patient population. Whereas in the past, multiple sclerosis diagnosis rested solely upon clinical criteria, current evaluation and treatment monitoring always include MRI. Sensitivity to changes in water content, the hallmark of MR, allows for detection of lesions missed by other imaging techniques. MRI also demonstrates lesion activity through the use of contrast administration and allows for more specific diagnosis of demyelinating lesions. MRI has altered the understanding of multiple sclerosis by revealing its dynamic nature, in which lesions progess and resolve undetected clinically.

The importance of multiple sclerosis and the central role played by MRI have conspired to generate myriad investigations directed at improving the contribution of MRI to evaluation and management. MR techniques applied to multiple sclerosis include magnetization transfer, proton spectroscopy, phosphorus spectroscopy, lesion volume quantitation, and diffusion anisotropy. Some of these techniques have already contributed to our understanding of multiple sclerosis and hold promise for more in the future. Despite these advances, protocols for basic clinical evaluation consist primarily of some form of $T_{2}$-weighted imaging, and, when appropriate, gadolinium enhancement.

\section{Use of fast FLAIR}

$T_{2}$-weighted imaging provides superior parenchymal contrast in brain imaging. However, small $T_{2}$-weighted hyperintense lesions may be obscured by adjacent, normally bright structures, most notably CSF. FLAIR (Fluid Attenuated Inversion Recovery) was developed to overcome this limitation through use of a magnetization preparation pulse applied prior to initiation of a $T_{2}$-weighted imaging sequence. Here, an inversion recovery pulse is applied and the spins are then allowed to relax back partially to the equilibrium state as defined by their $T_{1}$ characteristics. The imaging sequence is begun after a delay, $T_{\mathrm{I}}$ for inversion time, when the tissue to be suppressed is at the null point (effectively, with no net longitudinal magnetization) on its course back to equilibrium. In FLAIR the $T_{\mathrm{I}}$ is chosen so that CSF is at the null point. The end result is a predominantly $T_{2}$-weighted image with CSF suppressed through exploitation of its $T_{1}$ relaxation characteristics.

The $T_{1}$ of CSF is long, so a fairly long time (2000 to $2500 \mathrm{msec}$ ) must elapse between the inversion recovery pulse and the initiation of the imaging sequence. In early implementation, FLAIR was used with a spin echo sequence. Although the advantage of the technique was demonstrated, the imaging time (greater than $10 \mathrm{~min}$ ) was too long to gain widespread acceptance (Hajnal et al., 1992). More recently, FLAIR has been implemented with FSE/TSE sequence (fast FLAIR) yielding acceptable imaging times.

Considering the propensity of multiple sclerosis lesions to occur near ventricles, one might predict a sequence which suppresses the bright signal of CSF to uncover subtle periventricular lesions. Numerous studies have supported this expectation, and fast FLAIR is now widely regarded as providing superior sensitivity to supratentorial demyelinating lesions. Somewhat surprisingly, the same cannot be stated for infratentorial lesions. Several studies have demonstrated relative insensitivity of fast FLAIR in the posterior fossa (Bastianello et al., 1997; Tubridy et al., 1998). In light of this limitation, and recognizing the clinical importance of identifying posterior fossa lesions, a dual-echo $\mathrm{PD} / T_{2}$-weighted transverse sequence is included as a key component of the recommended protocol.

\section{Use of sagittal plane}

Evaluation of the corpus callosum in the sagittal plane enhances detection of subtle lesions and provides some specificity to the diagnosis of demyelinating disease relative to other periventricular white matter diseases (Gean-Marton et al., 1991; Jackson et al., 1993; Hashemi et al., 1995; Palmer et al., 1999). A scan in the sagittal plane is now expected by our referring clinicians whenever multiple scle- 
rosis is clinically suspected. Considering the advantage of fast FLAIR in evaluating periventricular disease, as well as its limitations in the brainstem, the sequence is applied to best advantage sagittally (Figure A5.1.1).

The choice of slice thickness for fast FLAIR sagittal evokes another discussion. With somewhat thicker slices (4 to $5 \mathrm{~mm}$ ), whole brain coverage is conveniently achieved. Although the focus of the scan is to evaluate the corpus callosum, complete brain coverage may uncover small subcortical lesions not readily apparent on the $\mathrm{PD} / T_{2}$-weighted transverse images. Use of thinner slices ( $3 \mathrm{~mm}$ or even $2 \mathrm{~mm}$ ) brings out subtle subependymal striations advocated by some as representing early demyelination which might not otherwise be detected (Hashemi et al., 1995). Use of such thin slices requires increasing the number of acquisitions to achieve reasonable image quality. In the author's experience, the potential benefit does not warrant the additional imaging time required.

\section{RARE (rapid acquisition and resolution enhancement) versus conventional spin echo}

Hennig et al. (1986) demonstrated a technique for diminishing scan time in which multiple spin echos were measured for a given excitation (that is, multiple lines of $k$-space) rather than one spin echo, as is the case with conventional spin echo. The train of spin echos was created through the use of a series of $180^{\circ}$ inversion pulses which were applied after the creation of each echo. Originally labeled RARE for Rapid Acquisition and Resolution Enhancement, the approach is now more commonly known by manufacturer labels-fast spin echo (FSE) and turbo spin echo (TSE). Although clearly faster, early implementations proved less sensitive in detection of demyelinating plaques. Following further evaluation, it became apparent that the problem resided not with the concept but rather with the long $T_{\mathrm{R}}$ values (4000 msec or greater) initially employed. With proper choice of parameters, TSE/FSE is very similar to the conventional spin echo method in lesion detection, while maintaining the advantage of reduced imaging time (Thorpe et al., 1994). Conventional spin echo imaging is comparable in lesion detection and so is left as an alternative to TSE/FSE in the recommended protocol.

\section{Augmenting enhancement}

Acute multiple sclerosis lesions are primarily inflammatory and carry with them a local disruption in the blood-brain barrier. Gadolinium enhancement on MRI provides a convenient marker for this disruption and correlates well with the presence of acute inflammation (Fig. A5.1.5; Nesbit et al., 1991; Katz et al., 1993). MRI thereby detects early events in the inflammatory process leading eventually to myelin loss and other tissue destruction (Trojano et al., 1996; Giovannoni et al., 1997).

Reflecting the importance of demonstrating lesion activity in multiple sclerosis, the efficacy of several techniques in improving detection of enhancement has been investigated. Techniques so investigated include triple-dose gadolinium, delayed imaging, and magnetization transfer (MT; see below) (Silver et al., 1997; Bastianello et al., 1998; Filippi et al., 1998a,b; Rovaris et al., 1998; Rovaris et al., 1999a,b). Of these techniques, triple-dose gadolinium is most effective, with several studies documenting significant increases ranging from $50 \%$ to $100 \%$ in the number of enhancing lesions detected. Delayed imaging and MT have some effect and may be particularly useful in combination. Each technique, of course, carries some penalty. For triple-dose gadolinium, the downside is simply one of increased cost. Delayed imaging alone is only mildly effective and of course requires additional time (20 min or more) for the delay (Silver et al., 1997). For MT, proper interpretation of the presence of enhancement necessitates performance of a pre-contrast MT scan. Some of these techniques are currently in use in clinical trials but are not, so far, part of standard clinical practice. They are therefore left as options.

\section{Contribution of magnetization transfer (MT)}

Protons in water may be detected using conventional MRI because they spin or precess at nearly the same frequency. These detectable protons are relatively "free" at the molecular level. A second population of protons exists, but for our purposes may only be detected indirectly. This second population consists of protons "bound" to complex macromolecules. The association with these macromolecules modulates the magnetic microenvironment of the "bound" protons, causing them to spin at varying frequencies. With this variation in frequency the protons cannot be directly measured. However, the magnetizations of the "bound" and "free" populations are not separate, and will exchange to some extent (Wolff and Balaban, 1989). Because of this exchange, manipulations of the signal of one group will be transferred to the other.
Miscellaneous Brain Pathology A5.1.11 
A

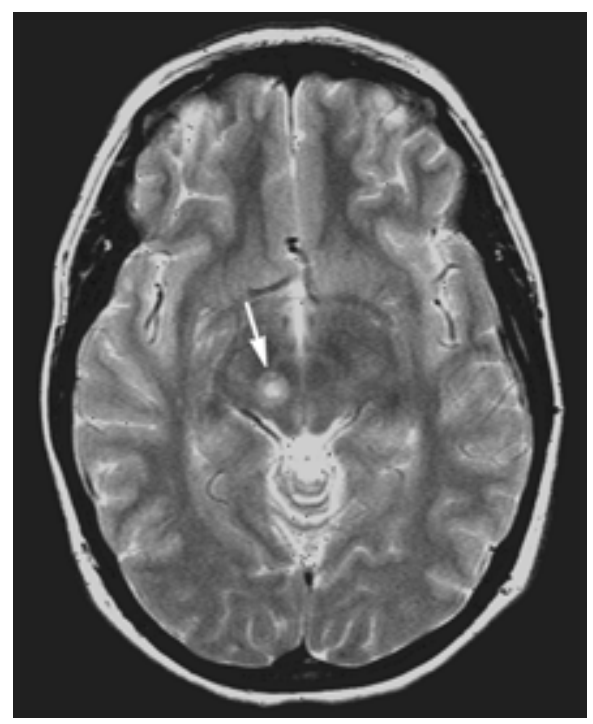

B

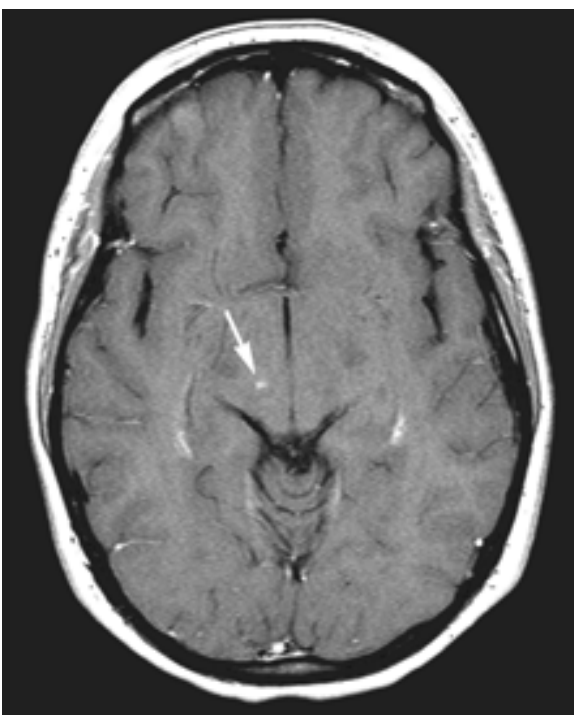

Figure A5.1.5 $T_{2}$-weighted TSE transverse (A) and post-contrast $T_{1}$-weighted spin echo transverse (B) images demonstrate typical enhancing demyelinating plaque. Contrast enhancement can be subtle in multiple sclerosis. Performance of $T_{2}$-weighted transverse sequence in identical location as post-contrast $T_{1}$-weighted transverse sequence aids recognition of enhancing lesions.

Several types of MT pulses exist. In the most conceptually straightforward approach, an offresonance saturation pulse is applied to the "bound" population. This saturation will be exchanged with the measurable "free" population, thereby partially decreasing their signal. The extent of the exchanged saturation depends on factors relating both to the saturation pulse and to the tissue to which it is applied. Tissues with higher number of "bound" protons (i.e., white matter) will be more effected by the magnetization pulse. Pathology, including demyelination, decreases the number of bound protons to be saturated and thereby diminishes the magnetization transfer effect. These effects provide several opportunities for application of MT to improve evaluation of white matter lesions. Such applications include but are not limited to: (1) improved sensitivity to enhancement, (2) improved specificity in diagnosis of multiple sclerosis plaques versus other periventricular white matter disease, and (3) further characterization of underlying pathology in multiple sclerosis plaques. Of these, in the author's opinion, the first two items are closest to becoming part of usual clinical practice and will be discussed below.

MT may be used to augment enhancement, predominantly by selectively suppressing nonenhancing structures. The application of
MT has been shown to approximately double the contrast-to-noise ratio of enhancing lesions compared with non-MT spin echo imaging (Finelli et al., 1994). The MT effect is not limited to enhancement, and nonenhancing lesions may exhibit increased signal on MT images. For this reason, precontrast MT imaging is needed to validly diagnose enhancement (Mehta et al., 1995).

MT may be helpful in distinguishing demyelinating lesions from periventricular white matter ischemic lesions. This follows from the increased MT effect in white matter ischemic lesions compared with demyelinating lesions of multiple sclerosis (Mehta et al., 1996). With the MT effect higher in ischemic white matter lesions, such lesions are suppressed along with normal brain, more so than demyelinating lesions. When inspecting MT $T_{1}$-weighted spin echo images, the difference translates into high signal in some demyelinating lesions, but the contrast between white matter and gray matter is suppressed (Figure A5.1.4). In general, multiple sclerosis is usually recognized by considering lesion distribution and morphology in conjunction with the clinical setting. In those cases in which such considerations are insufficient for confident diagnosis, MT may be a helpful option. 


\section{Critical Parameters and \\ Troubleshooting}

Motion artifacts may be a problem for nearly any MRI examination. Some motion may be present without significantly compromising the diagnostic utility of the examination. More frequent motion will be a problem. Proper care in placing and securing the patient in the head coil is critical, as is the interaction of the MRI technologist with the patient. In cases in which such measures are insufficient, prudent conscious sedation should be employed if at all possible. If sedation fails or is not possible, then faster imaging may be considered. As a first step, the provided sequences may be shortened simply by reducing the acquisition matrix $\left(N_{\mathrm{x}}\right.$, $N_{\mathrm{y}}$ ). Fast imaging techniques do exist and will be described in a later unit. However, such techniques are particularly limited in matters of contrast and are clearly inferior in evaluating white matter disease.

Proper contrast in fast FLAIR imaging is central to the recommended protocol. As noted previously, manufacturers vary in their implementation of this sequence, including the parameters employed. Utilization of FLAIR should begin with the manufacturer's standard parameters. Beyond this, some modifications may yield more appealing images with improved $T_{2}$-weighted lesion contrast. Use of a $T_{\mathrm{R}}$ that is too low (i.e., $<7000 \mathrm{msec}$ ) compromises both $T_{2}$-weighted contrast and CSF suppression. The $T_{\mathrm{E}}$ should be fairly long, at least 100 msec, again for reasons related to $T_{2}$-weighted contrast. The inversion time $\left(T_{\mathrm{I}}\right)$ is dictated by the $T_{1}$ of CSF and for a 1.5 Tesla magnet should be between $2100 \mathrm{msec}$ and $2400 \mathrm{msec}$. See Rydberg et al. (1995) for a thorough discussion of the considerations involved.

As already indicated, in early implementations of TSE/FSE, the $T_{\mathrm{R}}$ and $T_{\mathrm{E}}$ values chosen were too long to provide good $T_{2}$-weighted lesion contrast. $T_{\mathrm{R}}$ and $T_{\mathrm{E}}$ values should be chosen that are not longer than those given in Table A5.1.6. Use of longer relaxation parameters yields images which are visually acceptable; however fairly extensive experience proves that sensitivity to $T_{2}$-weighted hyperintense lesions is compromised.

Phase-encoding artifacts may occasionally be problematic in the posterior fossa on postcontrast images. Use of flow compensation usually solves such problems. Flow compensation requires a small increase in $T_{\mathrm{E}}$ (up to $\sim 17$ $\mathrm{msec}$ ). Such a parameter change does not diminish image quality. Alternatively, the addi- tion of a post-contrast $T_{1}$-weighted scan in a different plane, usually coronal, generally allows definitive interpretation of the presence of enhancement.

\section{Anticipated Results}

The exam should provide full imaging characterization of brain-parenchymal demyelinating lesions. To accomplish this end, effectively three types of sequences with various $T_{2}$ weighting are employed: fast FLAIR, proton density, and $T_{2}$. Each of these is performed in the plane in which it is most useful. Lesions of the corpus callosum, especially at the callosalseptal interface, are optimally visualized by FLAIR performed in the sagittal plane. Other periventricular lesions are well visualized on both the sagittal FLAIR and the $\mathrm{PD} / T_{2^{-}}$ weighted transverse images. Lesion activity, as manifested by blood-brain barrier compromise, will be demonstrated by the presence of enhancement.

The exam is not intended to optimally detect optic neuritis. Generally, this is not an important imaging indication as its diagnosis is clinically readily apparent and the MRI is usually employed to evaluate for the presence of other clinically occult lesions. If evaluation for optic neuritis is the clinical indication, then a dedicated orbit exam should be performed (see UNIT A7.5).

\section{Literature Cited}

Bastianello, S., Bozzao, A., Paolillo, A., Giugni, E., Gasperini, C., Koudriavtseva, T., Millefiorini, E., Horsfield, M.A., Colonnese, C., Toni, D., Fiorelli, M., Pozzilli, C., and Bozzao L. 1997. Fast spin-echo and fast fluid-attenuated inversion-recovery versus conventional spin-echo sequences for MR quantification of multiple sclerosis lesions. Am. J. Neuroradiol. 18:699-704.

Bastianello, S., Gasperini, C., Paolillo, A., Giugni, E., Ciccarelli, O., Sormani, M.P., Horsfield, M.A., Rovaris, M., Pozzilli, C., and Filippi, M. 1998. Sensitivity of enhanced MR in multiple sclerosis: Effects of contrast dose and magnetization transfer contrast. Am. J. Neuroradiol. 19:1863-1867.

Filippi, M., Rovaris, M., Capra, R., Gasperini, C., Yousry, T.A., Sormani, M.P., Prandini, F., Horsfield, M.A., Martinelli, V., Bastianello, S., Kuhne I, Pozzilli C, and Comi G. 1998a. A multi-centre longitudinal study comparing the sensitivity of monthly MRI after standard and triple dose gadolinium-DTPA for monitoring disease activity in multiple sclerosis. Implications for phase II clinical trials. Brain 121:2011-2020.

Filippi, M., Rovaris, M., Gasperini, C., Capra, R., Bastianello, S., Kuhne, I., and Yousry, T.A.
Miscellaneous Brain Pathology A5.1.13 
1998b. A preliminary study comparing the sensitivity of serial monthly enhanced MRI after standard and triple dose gadolinium-DTPA for monitoring disease activity in primary progressive multiple sclerosis. Journal of Neuroimaging 8:88-93.

Finelli, D.A., Hurst, G.C., Gullapali, R.P., and Bellon, E.M. 1994. Improved contrast of enhancing brain lesions on postgadolinium, $T_{1}$-weighted spin-echo images with use of magnetization transfer. Radiology 190:553-559.

Gean-Marton, A.D., Vezina, L.G., Marton, K.I., Stimac, G.K., Peyster, R.G., Taveras, J.M., and Davis, K.R. 1991. Abnormal corpus callosum: A sensitive and specific indicator of multiple sclerosis. Radiology 180:215-221.

Giovannoni, G., Lai, M., Thorpe, J., Kidd, D., Chamoun, V., Thompson, A J., Miller, D.H., Feldmann, M., and Thompson, E.J. 1997. Longitudinal study of soluble adhesion molecules in multiple sclerosis: correlation with gadolinium enhanced magnetic resonance imaging. Neurology 48:1557-1565.

Giugni, E., Pozzilli, C., Bastianello, S., Gasperini, C., Paolillo, A., Koudriavtseva, T., Frontoni, M., Farina, D., and Bozzao, L. 1997. MRI measures and their relations with clinical disability in relapsing-remitting and secondary progressive multiple sclerosis. Multiple Sclerosis 3:221-225.

Hajnal, J.V., Bryant, D.J., Kasuboski, L., Pattany, P.M., De Coene, B., Lewis, P.D., Pennock, J.M., Oatridge, A., Young, I.R., and Bydder, G. M. 1992. Use of fluid attenuated inversion recovery (FLAIR) pulse sequences in MRI of the brain. $J$. Comput. Assisted Tomogr. 16:841-844.

Hashemi, R.H., Bradley, W.G., Jr., Chen, D.Y., Jordan, J.E., Queralt, J.A., Cheng, A.E., and Henrie, J.N. 1995. Suspected multiple sclerosis: MR imaging with a thin-section fast FLAIR pulse sequence. Radiology 196:505-510.

Hennig, J., Nauerth, A., and Friedburg, H. 1986. RARE imaging: A fast imaging method for clinical MR. Magn. Reson. Med. 3:823-833.

Jackson, A., Fitzgerald, J.B., and Gillespie, J.E. 1993. The callosal-septal interface lesion in multiple sclerosis: Effect of sequence and imaging plane. Neuroradiology 35:573-577.

Katz, D., Taubenberger, J.K., Cannella, B., McFarlin, D.E., Raine, C.S., and McFarland, H.F. 1993. Correlation between magnetic resonance imaging findings and lesion development in chronic, active multiple sclerosis. Ann. Neurol. 34:661669.

Mehta, R.C., Pike, G.B., and Enzmann, D.R. 1995. Improved detection of enhancing and nonenhancing lesions of multiple sclerosis with magnetization transfer. Am. J. Neuroradiol. 16:17711778.

Mehta, R.C., Pike, G.B., and Enzmann, D.R. 1996. Measure of magnetization transfer in multiple sclerosis demyelinating plaques, white matter ischemic lesions, and edema. Am. J. Neuroradiol. 17:1051-1055.
Nesbit, G.M., Forbes, G.S., Scheithauer, B.W., Okazaki, H., and Rodriguez, M. 1991. Multiple sclerosis: Histopathologic and MR and/or CT correlation in 37 cases at biopsy and three cases at autopsy. Radiology 180:467-474.

Palmer, S., Bradley, W.G., Chen, D.Y., and Patel, S. 1999. Subcallosal striations: Early findings of multiple sclerosis on sagittal, thin-section, fast FLAIR MR images. Radiology 210:149-153.

Rovaris, M., Mastronardo, G., Gasperini, C., Prandini, F., Yousry, T.A., and Filippi, M. 1998. MRI evolution of new MS lesions enhancing after different doses of gadolinium. Acta Neurol. Scand. 98:90-93.

Rovaris, M., Mastronardo, G., Prandini, F., Bastianello, S., Comi, G., and Filippi, M. 1999a. Shortterm evolution of new multiple sclerosis lesions enhancing on standard and triple dose gadolinium-enhanced brain MRI scans. J. Neurol. Sci. 164:148-152.

Rovaris, M., Rodegher, M., Comi, G., and Filippi, M. 1999b. Correlation between MRI and shortterm clinical activity in multiple sclerosis: Comparison between standard- and triple-dose Gdenhanced MRI. Eur. Neurol. 41:123-127.

Rydberg, J.N., Riederer, S.J., Rydberg, C.H., and Jack, C.R. 1995. Contrast optimization of fluidattenuated inversion recovery (FLAIR) imaging. Magn. Reson. Med. 34:868-877.

Shellock, F.G. 1996. Pocket Guide to MR Procedures and Metallic Objects. Lippincott-Raven, Philadelphia.

Silver, N.C., Good, C.D., Barker, G.J., MacManus, D.G., Thompson, A.J., Moseley, I.F., McDonald, W.I., and Miller, D.H. 1997. Sensitivity of contrast enhanced MRI in multiple sclerosis. Effects of gadolinium dose, magnetization transfer contrast and delayed imaging. Brain 120:11491161.

Thorpe, J.W., Halpin, S.F., MacManus, D.G., Barker, G.J., Kendall, B.E., and Miller, D.H. 1994. A comparison between fast and conventional spin-echo in the detection of multiple sclerosis lesions. Neuroradiology 36:388-392.

Trojano, M., Avolio, C., Simone, I.L., Defazio, G., Manzari, C., De Robertis, F., Calo, A., and Livrea, P. 1996. Soluble intercellular adhesion molecule-1 in serum and cerebrospinal fluid of clinically active relapsing-remitting multiple sclerosis: correlation with Gd-DTPA magnetic resonance imaging-enhancement and cerebrospinal fluid findings. Neurology 47:1535-1541.

Truyen, L., Van Waesberghe, J.H., Van Walderveen, M.A., Van Oosten, B.W., Polman, C.H., Hommes, O.R., Ader, H.J., and Barkhof, F. 1996. Accumulation of hypointense lesions ("black holes") on $T_{1}$ spin-echo MRI correlates with disease progression in multiple sclerosis. $\mathrm{Neu}$ rology 47:1469-1476.

Tubridy, N., Barker, G.J., MacManus, D.G., Moseley, I.F., and Miller, D.H. 1998. Optimisation of unenhanced MRI for detection of lesions in multiple sclerosis: A comparison of five pulse se- 
quences with variable slice thickness. Neuroradiology 40:293-297.

van Walderveen, M.A., Barkhof, F., Hommes, O.R., Polman, C.H., Tobi, H., Frequin, S.T., and Valk, J. 1995. Correlating MRI and clinical disease activity in multiple sclerosis: Relevance of hypointense lesions on short- $T_{\mathrm{R}} /$ short- $T_{\mathrm{E}}\left(T_{1^{-}}\right.$ weighted) spin-echo images. Neurology 45:1684-1690.

Wolff, S.D., and Balaban, R.S. 1989. Magnetization transfer contrast (MTC) and tissue water proton relaxation in vivo. Magn. Reson. Med. 10:135144.
Key References

Shellock, 1996. See above.

Covers a number of important patient management issues related to MR imaging, including recommended safety procedures, a list of metallic implants that have been tested for MR compatibility, and a list of other sources on MR safety.

Contributed by Danial K. Hallam

University of Washington

Seattle, Washington 